\title{
Low-injection behaviour of a solar cell with a metallic grating back-reflector
}

\author{
Francisco Llopis, Mario M. Jakas \\ Departamento de Física Fundamental y Experimental, \\ Electrónica y Sistemas, Universidad de La Laguna \\ Avda. Astrofísico Francisco Sánchez, s/n \\ 38203 La Laguna, Spain \\ fllopis@ull.es, mmateo@ull.es
}

\author{
Ignacio Tobías \\ Instituto de Energía Solar \\ Universidad Politécnica de Madrid \\ Avda. Complutense, s/n \\ 28040 Madrid, Spain \\ tobias@ies-def.upm.es
}

\begin{abstract}
Recent studies have dealt with the possibility of increasing light absorption by using the so-called electric field enhancement taking place within the grooves of metallic gratings. In order to evaluate the potential improvements derived from the absorption increase, we employ a simplified model to analyze the low-injection behaviour of a solar cell with a metallic grating backreflector.
\end{abstract}

\section{INTRODUCTION}

The conversion efficiency of solar cells relies strongly on their absorption capability. The need of a higher absorbance is especially critical in intermediate band solar cells (IBSC). The first prototypes, fabricated with quantum-dot (QD) absorbers, exhibit low absorbance for infrared wavelengths. On the other hand, the performance of thin film silicon solar cells is also affected by incomplete absorption $[1,2]$.

Since absorption is proportional to the squared amplitude of the electric field, some recent studies have dealt with the possibility of taking advantage of resonance phenomena in the near field. These include surface plasmons in metal nanoparticles or confined resonances in metallic gratings $[3,4]$.

In this work we concentrate on the latter kind of phenomenon, given that the squared electric field inside the grating grooves may be up to two orders of magnitude larger than that of the incident light [4]. Accordingly, it is quite conceivable that this effect may have noticeable consequences on photovoltaic devices. Therefore, in this work we outline a relatively simple method to asses the impact of using a diffraction grating as a back reflector in a solar cell. This can be accomplished in two steps: (1) evaluation of the optical generation rate profiles inside the device and, (2) evaluation of the minority-carrier excess concentration within the cell.

\section{The Enhancement Of The Electric Field}

The device under consideration includes a metallic grating back reflector (figure 1). A TE polarized wave with wavelength $\lambda$ illuminates the whole structure, which is comprised by the grating itself and the surrounding medium, whose refractive index is $v_{l}$. Each groove has a depth $h$, a spacing $d$, and a width c. As pointed before, the electric field can be enhanced inside the grooves of the grating. In order to ascertain the potential improvements derived from this situation, the optical generation rate, and hence the squared amplitude of the fields, must be known in the first place. To this end, the electric field inside the grooves $(y<0)$ and above the top of a perfectly conducting grating $(y>0)$ can be calculated using the Rayleigh-Bloch method $[5,6]$.

For normal incidence, the incident field reads

$$
E_{z i}(x, y)=\exp \left(-i \kappa \nu_{1} y\right)
$$

$\kappa=2 \pi / \lambda$ being the vacuum wavenumber. As a result of the interaction between $E_{z i}$ and the grating, an electric field is scattered off the grating, which can be expressed as:

$$
E(x, y)=E_{z i}(x, y)+\sum_{n=-\infty}^{\infty} A_{n} \exp \left[i\left(\alpha_{n} x+\chi_{n} y\right)\right] \quad(y>0),
$$

where the $n$-th reflected wave has an amplitude $A_{n}$ and wavevector components

$$
\alpha_{n}=\frac{2 \pi}{d} n, \quad \chi_{n}=\sqrt{\kappa^{2} v_{1}^{2}-\alpha_{n}^{2}} .
$$

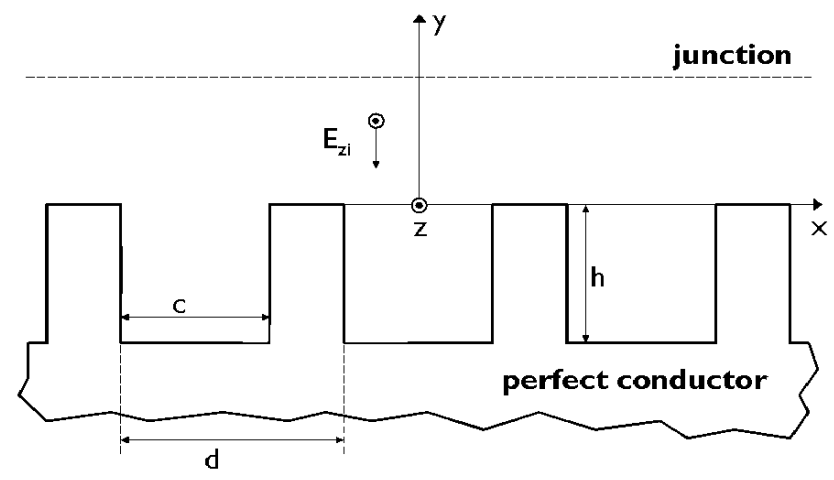

Figure 1. Perfectly conducting grating illuminated by TE polarized light 
The interaction also results in an electric field inside the grooves that can be expressed by the modal expansion

$$
E(x, y)=\sum_{m=1}^{\infty} a_{m} \phi_{m}(x, y) \quad(y<0)
$$

The modal functions $\phi_{m}$ in (4) read:

$$
\phi_{m}(x, y)=\sin \left[\mu_{m}(y+h)\right] \sin (m \pi x / c)
$$

if $m$ is even, and

$$
\phi_{m}(x, y)=\sin \left[\mu_{m}(y+h)\right] \cos (m \pi x / c),
$$

if $m$ is odd, with $\mu_{m}$ given by

$$
\mu_{m}=\left[\left(\kappa \nu_{1}\right)^{2}-(m \pi / c)^{2}\right]^{1 / 2} .
$$

Let us assume that the electric field inside the grooves is dominated by the fundamental mode ( $m=1)$, having the remaining modes an imaginary $\mu_{m}$ spatial frequency and negligible amplitude. This condition is achieved for wavelengths $\lambda$ such that $c v_{1}<\lambda<2 c v_{1}$. Furthermore, when the condition $\lambda>d v_{1}$ holds, the dominant reflected orders are the specular one ( 0 -th order), and \pm 1 -st orders are evanescent Under these assumptions, Wirgin and Maradudin [6] find that:

(1) The TE electric field peaks are located near the quasiresonant wavelengths

$$
\lambda_{j}=2 v_{1}\left(j^{2} / c^{2}+1 / h^{2}\right)^{-1 / 2} .
$$

(2) The magnitude of the electric field inside the grooves at the wavelength $\lambda_{\text {j }}$ can be expressed as

$$
|E(0, y)|_{\lambda_{j}=\lambda_{j}}=\frac{8 \chi_{0}}{\pi}\left|\frac{\sin \left[\mu_{1}\left(\lambda_{j}\right) \cdot(y+h)\right]}{\mu_{1}\left(\lambda_{j}\right)}\right| .
$$

For a given value of $y$, the electric field magnitude reaches its absolute maximum at $\mu_{i}=0$. The magnitude will be larger the smaller is $\mu_{1}$. This, in turn, implies to choose $h$ as large as possible. In the limit $h \rightarrow \infty$ we find $\lambda_{j}=2 \nu_{1} c$.

Thus, if the $h / c$ ratio is large enough, we can employ this result to estimate the dimensions of a grating for a given resonance wavelength $\lambda_{r}$. For example, QD layers designed for AsGa IB solar cells are sensitive to photon energies close to 0.3 $\mathrm{eV}(4.1 \mu \mathrm{m})$

After substituting $v_{1}=3.6$ and $\lambda_{r}=4.1 \mu \mathrm{m}$ in the expression $c=\lambda_{r} / 2 v_{l}$, we obtain $c=0.57 \mu \mathrm{m}$. By further assuming $d=1.2 c$, and a given $h / c$ aspect ratio $(2,3$ or 4$)$, we have employed the Rayleigh-Bloch method to calculate the modal and reflected orders amplitudes introduced in (2) and (4). Results of the electric field so calculated right at the centre of the groove are shown in figure 2. As one can readily see, the electric field becomes substantially increased in the vicinity of certain photon energies. The main peaks are also located near our target $(0.3 \mathrm{eV})$.

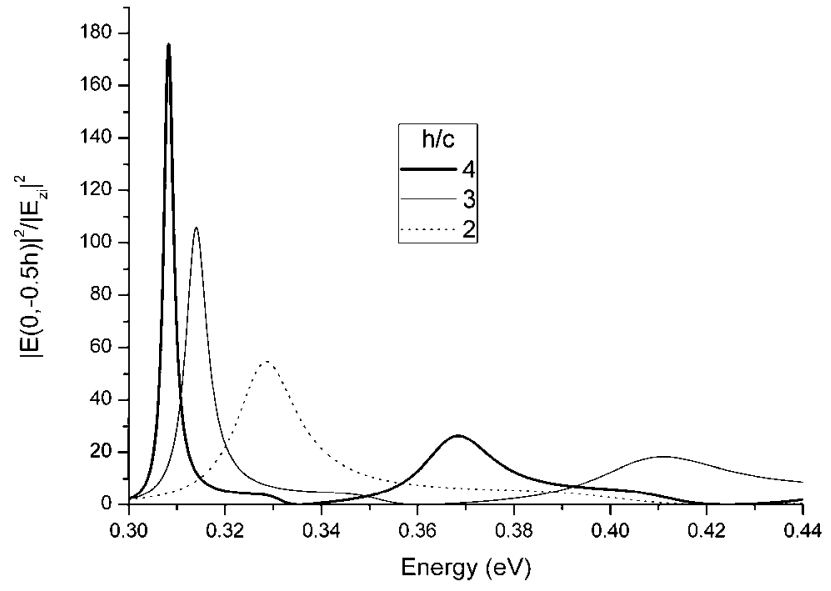

Figure 2. Electric field enhancement at the position $(0,-0.5 \mathrm{~h})$ inside the groove as a function of the photon energy, for TE polarized light incident perpendicular to the grating, and $h / c=2,3$ and 4 aspect ratio

When the device is illuminated by sunlight, we must average the squared amplitudes of the TE and TM electric fields to correctly evaluate the generation rate inside the grooves. However, the condition stated before does not guarantee the simultaneous enhancement of the TE and TM electric fields [4]. Therefore, if we neglect the contribution due to the TM polarization, the squared magnitude of the electric field inside the grooves can be approximated by

$$
|E(x, y)|^{2} \cong \frac{1}{2}\left|a_{1}\right|^{2} \sin ^{2}\left[\mu_{1}(y+h)\right] \cos ^{2}(\pi x / c),
$$

where we have also assumed that the fields are dominated by the fundamental mode contribution. Of course, the latter assumption applies whenever the geometrical features are chosen according the conditions stated above. Otherwise, a greater number of modal terms should be retained to calculate the internal electric fields.

\section{CAlCulation OF THE EXCESS MinORITy CARRIER CONCENTRATION}

In order to assess the performance of the devised structure in a photovoltaic cell, we must calculate the excess minority carrier density. Thus, first we need to calculate the electronhole generation.

We will assume that the dielectric surrounding the metallic reflector is a $n$-type semiconductor, and that the device junction is placed at $y=y_{j}$ (figure 3). Since the generation rate is the ratio between the energy absorption and the photon energy $E_{p h}$, we have

$$
G(x, y, \lambda)=\frac{\alpha(\lambda) \sqrt{\frac{\varepsilon}{\mu_{0}}}|E(x, y, \lambda)|^{2}}{E_{p h}(\lambda)}
$$


$\alpha$ being the absorption coefficient. As discussed in the previous section, the metallic grating reflector can enlarge the electric field magnitude in the vicinity of a resonant wavelength. Thus, in $(11)|E(x, y, \lambda)|^{2}$ includes the effect of the electric field enhancement as calculated before.

In this work we assume that the QD arrays are allocated inside the grooves $\left(R_{2}\right.$ region). Therefore, the generation rate (11) will be only calculated within this region, thus allowing us to concentrate on the role played by the QD arrays. The contribution of the $p$ region to the device current density will also be neglected.

As is well known, if the electric field in the neutral $n$ region of a $p n$ junction device is neglected, the equation for the excess minority hole reads [7]:

$$
\nabla^{2} p_{n}^{\prime}-\frac{1}{L_{p}^{2}} p_{n}^{\prime}=-\frac{G}{D_{p}}
$$

where $p_{n}^{\prime}, L_{p}$ and $D_{p}$ are the excess concentration, diffusion length and diffusion constant of holes, respectively.

Equation (12) can be numerically solved by employing the finite element method [8]. Regarding the boundary conditions, since the analysis is restricted to the cell shown in figure 3 , the normal derivative of the solution must vanish along the lines $\Gamma_{2}$ and $\Gamma_{3}$. If surface recombination at metal-semiconductor interface is neglected the same condition can be imposed on $\Gamma_{4}$. Finally, when the device operates in short-circuit the solution vanishes at the junction.

Once the carrier excess distribution is known, the shortcircuit current density contribution of the cell shown in figure 3 can be readily estimated from

$$
J_{s c}(\lambda)=\frac{q \int_{R_{1} \cup k_{2}}\left[G_{p}(x, y, \lambda)-U_{p}(x, y, \lambda)\right] d x d y}{d},
$$

where the recombination rate is given by

$$
U_{p}(x, y, \lambda)=\frac{p_{n}^{\prime}}{\tau_{p}}
$$

\section{Results ANd Discusion}

The short-circuit current of the cell shown in figure 3 can be compared to that exhibited by the same cell when a flat reflector is used instead the grating.

The groove width, height and spacing of the grating cell of figure 3 have been set as $c=0.57 \mu \mathrm{m}, h=3 c$ and $d=1.2 c$, respectively. When the flat reflector replaces the grating, the bottom layer width has been assumed to be $h$. In both cases, the width of the top layer is $y_{j}=0.5 \mu \mathrm{m}$.

The remaining parameters are: $D_{p}=7.5 \mathrm{~cm}^{2} \mathrm{~s}^{-1}, v_{l}=3.6$ and $\alpha=0.1 \mathrm{~cm}^{-1}$. The hole lifetime is assumed to be $\tau_{p}=10 \mathrm{~ns}$ in $R_{l}$, but $\tau_{p}=1 \mathrm{~ns}$ in $R_{2}$. The same applies to the top and bottom layers of the cell with flat reflector.

The ratio between the short-circuit current of the cell with grating reflector $\left(J_{S C, D G}\right)$ and that of the cell with flat reflector
$\left(J_{S C, F R}\right)$ is plotted in figure 3 . As one can see, $J_{S C, D G} / J_{S C, F R}$ may be as large as seven at $0.314 \mathrm{eV}$, in a good agreement with that observed for the electric field.

\section{CONCLUSIONS}

In this work we have presented a preliminary version of the low-injection model and, as an example, the results expected for a given set of parameters. In this stage, a full parametric analysis is outside the scope of this work. Such an analysis, as well as extending the model to include other effects such as the surface recombination and carrier transport in the depletion region, is the objective of the forthcoming paper.

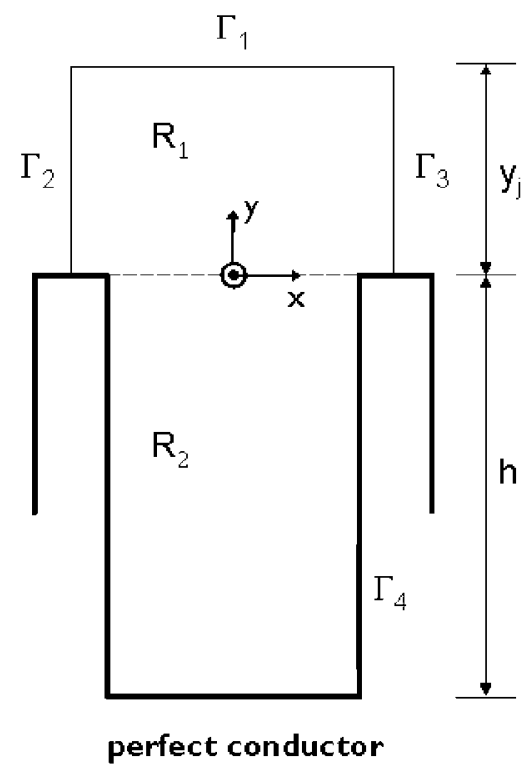

Figure 3. Two-dimensional cell considered in the low-injection model

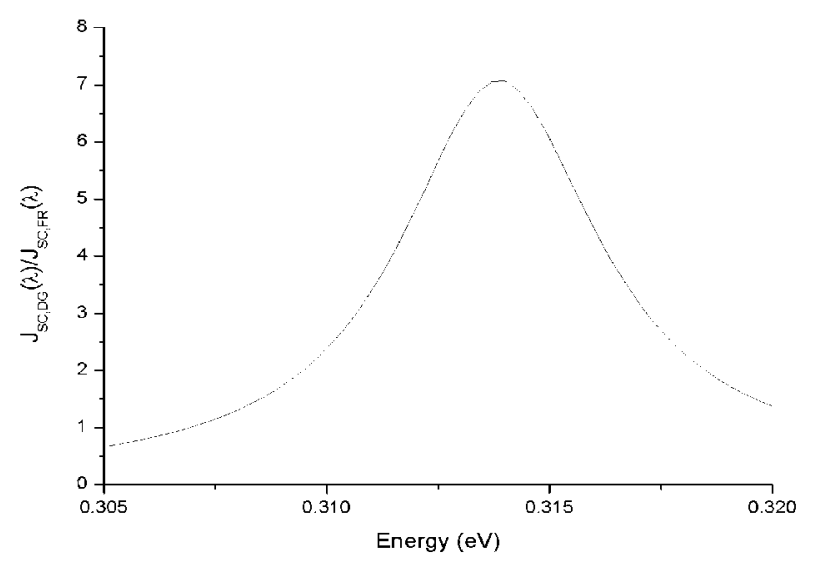

Figure 4. Ratio between the short-circuit current densities $J_{S C . D Y Y}$ (cell with grating reflector) and $J_{S C . F R}$ (cell with flat reflector). (See text for details) 


\section{ACKNOWLEDGMENT}

This work has been supported in part by the IBPOWER Grant 211640 of the European Commission and the NUMANCIA grant S-0505/ENE/0310 of the Comunidad de Madrid.

\section{REFERENCES}

[1] A. Luque and A. Martí, "The Intermediate Band Solar Cell: Progress Toward the Realization of an Attractive Concept", Adv. Mater., vol. 21, pp. $1-15,2010$.

[2] B. Sopori, "Thin film silicon solar cells", in Handbook of Photovoltaic Science and Technology, A. Luque and S. Hegedus (eds), pp. 307-358, Wiley, 2004.
[3] M. J. Mendes, A. Luque, I. Tobías, and A. Martí, "Plasmonic light enhancement in the near-field of metallic nanospheroids for application in intermediate band solar cells," Appl. Phys. Lett., vol. 95, pp. 3, 2009.

[4] F. Llopis, I. Tobías, and M.M. Jakas, "Light intensity enhancement inside the grooves of metallic gratings", J. Opt. Soc. Amer. B, vol. 27, pp. $1998-2006,2010$.

[5] J. R. Andrewartha, J. R. Fox, and I. J. Wilson, "Resonance anomalies in the lamellar grating", Optica Acta, vol. 26, pp. 69-89, 1979.

[6] A. Wirgin and A. A. Maradudin, "Resonant enhancement of the electric field in the grooves of bare metallic gratings exposed to S-polarized light", Phys. Rev. B, vol. 31, pp. 5573-5576, 1985.

[7] S.M. Sze, "Physics of Semiconductor Devices", John Wiley \& Sons, 1981.

[8] O.C. Zienkiewicz and R.L. Taylor. "The Finite Element Method", McGraw-Hill, 1994. 